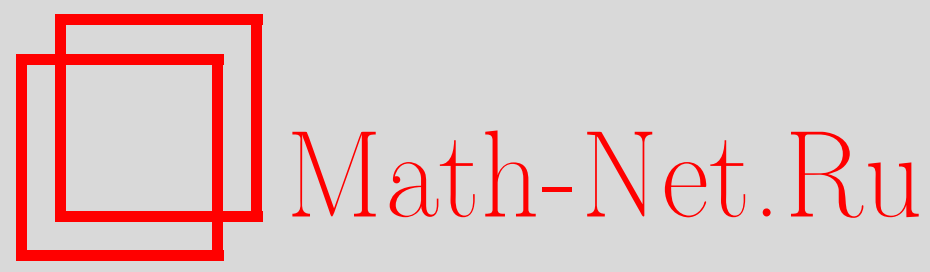

Т. Я. Азизов, А. И. Барсуков, Алгебраическая структура $H$ диссипативных операторов в конечномерном пространстве, Матем. заметки, 1998, том 63, выпуск 2, 163-169

DOI: https://doi.org/10.4213/mzm1263

Использование Общероссийского математического портала Math-Net.Ru подразумевает, что вы прочитали и согласны с пользовательским соглашением http://www.mathnet.ru/rus/agreement

Параметры загрузки:

IP : 52.6 .47 .48

26 апреля 2023 г., 12:11:06

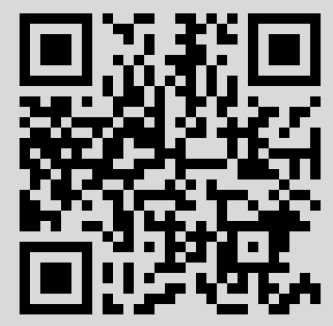




\title{
АЛГЕБРАИЧЕСКАЯ СТРУКТУРА $H$-ДИССИПАТИВНЫХ ОПЕРАТОРОВ В КОНЕЧНОМЕРНОМ ПРОСТРАНСТВЕ
}

\author{
Т. Я. Азизов, А. И. Барсуков
}

\begin{abstract}
Исследуются свойства жордановых представлений $H$-диссипативных операторов в конечномерном индефинитном $H$-пространстве. Приведено алгебраическое доказательство существования у таких операторов максимальных семидефинитньх инвариантных подпространств.

Библиография: 14 названий.
\end{abstract}

Введение. Пусть $\mathscr{E}^{n}-n$-мерное комплексное гильбертово пространство со скалярным произведением $(\cdot, \cdot), H: \mathscr{E}^{n} \rightarrow \mathscr{E}^{n}$ - самосопряженньй обратимый оператор. Полуторалинейная, вообще говоря, индефинитная форма $[\cdot, \cdot]=(H \cdot, \cdot)$ назьвается H-метрикой.

Напомним некоторые определения (подробнее см., например, [1]). Естественным образом, в соответствии со знаком $H$-метрики, вводится знак вектора и подпространства. Так, например, вектор $x$ назьвается положительным, если $[x, x]>0$, а подпространство $\mathscr{L}$ - нейтральным, если $[x, x]=0$ при всех $x \in \mathscr{L}$, и т.д. Подпространство

$$
\mathscr{L}^{\perp}=\left\{x \in \mathscr{E}^{n} \mid[x, y]=0 \text { при всех } y \in \mathscr{L}\right\}
$$

назьвается $H$-ортогональным дополнением подпространства $\mathscr{L}$, а $\mathscr{L}^{0}=\mathscr{L} \cap \mathscr{L}^{\perp}-$ его изотропной частью. Если $\mathscr{L}^{0}=\{\theta\}$, то $\mathscr{L}$ называется невырожденным подпространством, в противном случае-вырожденным, авекторы $x \in \mathscr{L}^{0}-$ изотропными в $\mathscr{L}\left(\right.$ и в $\left.\mathscr{L}^{\perp}\right)$. Заметим, что если $\mathscr{L}^{0} \neq\{\theta\}$, то каждьй вектор $x \in \mathscr{L}^{0}$ нейтрален; каждый нейтральньй вектор подпространства $\mathscr{L}$ изотропен тогда и только тогда, когда $\mathscr{L}$ семидефинитно, т.е. неотрицательно или неположительно.

Оператор $A^{*}: \mathscr{E}^{n} \rightarrow \mathscr{E}^{n}$ назьвается $H$-сопряженным , если $[A x, y]=\left[x, A^{*} y\right]$ при всех $x, y \in \mathscr{E}^{n}$. Так же, как в случае гильбертова пространства, определяются и другие классы операторов. Так, например, оператор $A$ называется $H$-диссипативным, если $\operatorname{Im}[A x, x] \geqslant 0, x \in H$, и $H$-самосопряжсенным, если $\operatorname{Im}[A x, x]=0, x \in H$, т.е. $[A x, y]=[x, A y]$.

Базис

$$
\left\{e_{i, j_{i}}\right\}, \quad j_{i}=1,2, \ldots, n_{i}, \quad i=1,2, \ldots, k, \quad \sum_{i=1}^{k} n_{i}=n
$$

Работа первого автора выполнена при поддержке фонда INTAS, грант № 93-0249. Работа второго автора выполнена при поддержке Международного научного фонда и правительства России, грант NZP300. 
назьвается $A$-жсордановым, если каждый из наборов $\left\{e_{i, j_{i}}\right\}_{j_{i}=1}^{n_{i}}, i=1,2, \ldots, k$, является жордановой цепочкой оператора $A$, т.е. каждому указанному набору соответствует собственное значение $\lambda_{i} \in \sigma_{p}(A)$ оператора $A$ такое, что

$$
\left(A-\lambda_{i} I\right)^{p} e_{i, n_{i}}=e_{i, n_{i}-p}, \quad p=0,1, \ldots, n_{i}, \quad e_{i, 0}=\theta, \quad i=1,2, \ldots, k .
$$

Статья посвящена исследованию свойств $H$-диссипативных операторов $A$ в конечномерном пространстве. Показано существование $A$-жорданова базиса (1) такого, что линейная оболочка каждого из наборов $\left\{e_{i, j_{i}}\right\}_{j_{i}=1}^{n_{i}}, i=1,2, \ldots, k$, является невырожденньп подпространством. Основным результатом работы является доказательство невырожденности для любого базиса каждой линейной оболочки наборов $\left\{e_{i, j_{i}}\right\}_{j_{i}=1}^{n_{i}}$, $i=1,2, \ldots, k$, равной длины.

Вопрос существования для случая $H$-самосопряженного оператора $A$ рассматривался ранее и даже вошел в монографии [2], [3]. Для несамосопряженного оператора это более трудная задача, и здесь, как правило, доказьвается существование биортогональной “сопряженной” системы. Этой проблеме для $H$-диссипативных операторов посвящен ряд работ (см. [4] и библиографию к ней), а для произвольных матриц - работа [5]. Кроме того, в [6]-[8] подобный вопрос решен в более общем случае операторного пучка.

Вопрос о том, что для любого базиса каждая линейная оболочка наборов $\left\{e_{i, j_{i}}\right\}_{j_{i}=1}^{n_{i}}$, $i=1,2, \ldots, k$, равной длины является невырожденным подпространством в случае $H$-самосопряженного оператора был, по-видимому, впервые поставлен и решен в [9], где существенно использовалось свойство самосопряженности. В настоящей работе найден другой подход, не использующий это обстоятельство.

Описанные вьше утверждения применены нами, как иллюстрация, к “конечномерному" доказательству известного результата [10], [11] о существовании максимального семидефинитного $A$-инвариантного подпространства (другие подходы в этом направлении см. в [4], [12]). Близкие результаты для $H$-сжатий, связанных, как известно, с $H$-диссипативными операторами преобразованием Кэли-Неймана, можно найти в [13].

1. Структура невырожденных инвариантных подпространств $H$-диссипативных операторов. Пусть сперва спектр $\sigma(A) H$-диссипативного оператора $A$ состоит из единственной точки $\lambda=0$. Докажем две ключевые леммы.

Лемма 1. Если вектор $e_{s} \in \mathscr{E}^{n}$ таков, что $A^{s-1} e_{s} \neq \theta, A^{s} e_{s}=\theta$, то существует вектор $f_{s} \in \mathscr{E}^{n}$ такой, что

$$
A^{s-1} e_{s}=A^{* s-1} f_{s} \quad u \quad A^{* s} f_{s}=\theta .
$$

ДоказАтЕльство. Из [14] (см. также [1, с. 125]) следует, что если $A$ есть $H$-диссипативньй оператор и для некоторого $e \in \mathscr{E}^{n}$ и натурального $m$ имеем $A^{m} e=\theta$, то

$$
\begin{gathered}
A^{* m-[m / 2]} A^{[m / 2]} e=\theta, \\
A^{* m-[m / 2]-1} A^{[m / 2]} e=A^{m-1} e .
\end{gathered}
$$

Пусть $x \in \operatorname{ker} A^{s-1}$. Используя (4), получим

$$
\left[A^{s-1} e_{s}, x\right]=\left[A^{* s-[s / 2]-1} A^{[s / 2]} e_{s}, x\right]=\left[e_{s}, A^{*[s / 2]} A^{s-[s / 2]-1} x\right] .
$$


Из тождества $[s / 2]+[(s-1) / 2]=s-1$ и соотношения $(3)$ следует

$$
\left[A^{s-1} e_{s}, x\right]=\left[e_{s}, A^{* s-[(s-1) / 2]-1} A^{[(s-1) / 2]} x\right]=0 .
$$

Следовательно, вектор $A^{s-1} e_{s} H$-ортогонален ядру $\operatorname{ker} A^{s-1}$, что влечет включение $A^{s-1} e_{s} \in \operatorname{ran} A^{* s-1}$, т.е. существует вектор $f_{s} \in \mathscr{E}^{n}$ такой, что $A^{s-1} e_{s}=A^{* s-1} f_{s}$. Поскольку для $H$-диссипативного оператора $A: \mathscr{E}^{n} \rightarrow \mathscr{E}^{n}$ справедливо равенство (см. $[14],[1$, c. 121])

$$
\operatorname{ker} A=\operatorname{ker} A^{*}
$$

имеем $A^{* s} f_{s}=\theta$.

Лемма 2. Инвариантное подпространство $\mathscr{L}$ оператора $А$ невырохсдно тогда и только тогда, когда $\mathscr{L} \cap \mathscr{L}^{\perp} \cap \operatorname{ker} A=\{\theta\}$.

ДокАЗАТЕльство. В одну сторону утверждение доказывается тривиально: если $\mathscr{L}$ невырождено, т.е. $\mathscr{L}^{0}=\mathscr{L} \cap \mathscr{L}^{\perp}=\{\theta\}$, то и $\mathscr{L} \cap \mathscr{L}^{\perp} \cap \operatorname{ker} A=\{\theta\}$.

Для доказательства обратной импликации достаточно проверить, что подпространство $\mathscr{L}^{0}$ инвариантно относительно $A$, и воспользоваться тем, что $\sigma(A)=\{0\}$. В силу $A$-инвариантности $\mathscr{L}$ получаем

$$
\left[A^{*} x, y\right]=0 \quad \text { при всех } x \in \mathscr{L}^{0}, y \in \mathscr{L} .
$$

Из определения $H$-диссипативного оператора следует, что и оператор $-A^{*} H$-диссипативен. Поэтому равенство (6) при $y=x$ влечет $A^{*} x=A x$, т.е. $\mathscr{L}^{0} A$-инвариантно.

Зафиксируем в $\mathscr{E}^{n} A$-жорданов базис (1). Предположим

$$
\begin{aligned}
k_{1} & :=n_{1}=n_{2}=\cdots=n_{l_{1}}>k_{2}:=n_{l_{1}+1}=n_{l_{1}+2}=\cdots=n_{l_{2}}>\cdots \\
& >k_{s}:=n_{l_{s-1}+1}=n_{l_{s-1}+2}=\cdots=n_{k} .
\end{aligned}
$$

Каждому подмножеству $\mathbf{S} \subset\{1,2, \ldots, s\}$ поставим в соответствие подпространство

$$
\mathscr{L}_{\mathbf{S}}=\text { l.h. }\left\{\mathscr{L}_{r} \mid r \in \mathbf{S}\right\}
$$

где $\mathscr{L}_{r}=$ l.h. $\left\{e_{i, j_{i}} \mid j_{i}=1,2, \ldots, k_{r}, i=n_{l_{r-1}+1}, \ldots, n_{l_{r}}\right\}, r=1,2, \ldots, s, n_{l_{0}+1}=n_{1}$, $n_{l_{s}}=n_{k}$.

ЛЕмма 3. При каждом $\mathbf{S} \subset\{1,2, \ldots, s\}$ подпространство $\mathscr{L}_{\mathbf{S}}$ невырожсдено.

ДокАЗАТЕЛЬСтво. Поскольку $\mathscr{L}_{\mathrm{S}}$ есть $A$-инвариантное подпространство, в силу леммы 2 достаточно проверить, что $\mathscr{L}_{\mathrm{S}} \cap \mathscr{L}_{\mathrm{S}}^{\perp} \cap \operatorname{ker} A=\{\theta\}$.

Пусть $x_{0} \in \mathscr{L}_{\mathbf{S}} \cap \mathscr{L}_{\mathrm{S}}^{\perp} \cap \operatorname{ker} A$. Как нетрудно проверить, такой вектор представляется в виде

$$
x_{0}=\sum_{r \in \mathrm{S}} \sum_{\left\{i \mid n_{i}=k_{r}\right\}} \alpha_{i} e_{i, 1},
$$

или, что эквивалентно,

$$
x_{0}=\sum_{r \in \mathrm{S}} \sum_{\left\{i \mid n_{i}=k_{r}\right\}} \alpha_{i} A^{n_{i}-1} e_{i, n_{i}}
$$


Проверим, что $x_{0} H$-ортогонален $\operatorname{ker} A^{k_{j}}$, где $j_{0}=\max \{i \in \mathbf{S}\}$. В силу изотропности этого вектора в $\mathscr{L}_{\mathrm{S}}$ достаточно показать, что $x_{0} H$-ортогонален l.h. $\left\{e_{i, 1} \mid n_{i} \neq k_{r}\right.$, $r \in \mathbf{S}\}$.

Рассмотрим следующие три случая.

1) Пусть $j<j_{1}=\min \{i \in \mathbf{S}\}$. Из определения (7) следует $k_{j}>k_{j_{0}}>k_{j_{1}}$. Поэтому для любого $z \in \mathscr{L}_{k_{j}} \cap \operatorname{ker} A^{k_{j_{0}}}$ имеет место представление $z=A y$, где $y \in \mathscr{L}_{k_{j}}$. Равенство (5) влечет

$$
\left[x_{0}, z\right]=\left[x_{0}, A y\right]=\left[A^{*} x_{0}, y\right]=0 .
$$

2) Пусть $j>j_{0}$, т.е. $k_{j}<k_{j_{1}}<k_{j_{0}}$. Без ограничения общности будем считать, что вектор (9) принадлежит $\operatorname{ran} A^{k_{j_{0}}-1}$ и не принадлежит $\operatorname{ran} A^{k_{j_{0}}}$, т.е. жорданова цепочка $x_{0}=A^{k_{j_{0}}-1} y_{0}, \ldots, y_{0}$ имеет длину $k_{j_{0}}$. В силу леммы 1 сушествует вектор $f_{0}$ такой, что $x_{0}=A^{* k_{j_{0}}{ }^{-1}} f_{0}$. Теперь $H$-ортогональность $x_{0}$ к $w \in \mathscr{L}_{j} \cap \operatorname{ker} A^{k_{j}}$ следует из равенств $\left[x_{0}, w\right]=\left[A^{* k_{j_{0}}-1} f_{0}, w\right]=\left[f_{0}, A^{k_{j_{0}}-1} w\right]=0$.

Если $j_{0}=j_{1}$, то $x_{0} H$-ортогонален $\operatorname{ker} A^{k_{j_{0}}}$. Если же $j_{0} \neq j_{1}$, то следует рассмотреть и следующий возможньй случай.

3) Пусть $j_{1}<j<j_{0}$, т.е. $k_{j_{0}}<k_{j}<k_{j_{1}}$.

Из случаев 1) и 2) в силу представления

$$
x_{0}=x_{1}+x_{2}, \quad x_{1} \in \text { l.h. }\left\{\mathscr{L}_{i} \mid i \in \mathbf{S}, j_{1} \leqslant i<j\right\}, \quad x_{2} \in \text { l.h. }\left\{\mathscr{L}_{i} \mid i \in \mathbf{S}, j<i \leqslant j_{0}\right\},
$$

следует $H$-ортогональность $x_{0} \mathrm{~K} v \in \mathscr{L}_{j} \cap \operatorname{ker} A^{k_{j_{0}}}$.

Таким образом, $x_{0} H$-ортогонален $\operatorname{ker} A^{k_{0}}$, поэтому $x_{0} \in \operatorname{ran} A^{* k_{j_{0}}}$, т.е. существует вектор $g_{0}$ такой, что $x_{0}=A^{* k_{j_{0}}} g_{0}$. Из равенства (2), меняя ролями $A$ и $-A^{*}$ и заменяя $s-1$ на $k_{j_{0}}$, получим, что $x \in \operatorname{ran} A^{k_{j_{0}}}$. Это противоречит предположению $x \notin \operatorname{ran} A^{k_{j_{0}}}$ в случае 2 ).

Вернемся к общему случаю. Пусть $H$-диссипативньй оператор $A: \mathscr{E}^{n} \rightarrow \mathscr{E}^{n}$ имеет $m \leqslant n$ различных вещественных собственных значений $\left\{\lambda_{i}\right\}_{i=1}^{m}$. Вновь зафиксируем $A$-жорданов базис $(1)$. Символом $\mathscr{L}_{\lambda, \mathrm{S}(\lambda)}$ обозначим подпространство $\mathscr{L}_{\mathrm{S}(\lambda)}$, соответствующее оператору $A-\lambda I$ согласно формуле (8).

Tеорема 1. Подпространство $\mathscr{M}=1 . h .\left\{\mathscr{L}_{\lambda, \mathrm{S}(\lambda)} \mid \lambda \in \sigma(A)\right\}$ невырохсдено.

ДокАЗАтЕЛЬСтво. Допустим противное: подпространство $\mathscr{M}$ вырождено. Тогда согласно лемме 2 его изотропная часть содержит хотя бы один собственньй вектор $x_{0} \neq \theta$ оператора $A$. Без ограничения общности можно считать, что он соответствует собственному значению $\lambda=0$. Пусть $\mu$-собственное значение оператора $A$, а $\mathscr{L}(\mu)$ - соответствующее корневое подпространство. Из леммы 3 следует, что $x_{0} H$-ортогонален $\mathscr{L}(0)$. Покажем, что $x_{0} H$-ортогонален $\mathscr{L}(\mu), \mu \neq 0$. Если $s$ - длина максимальной жордановой цепочки, соответствующей собственному значению $\mu$, то

$$
\left[\left(A^{*}-\mu I\right)^{s} x_{0}, y\right]=\left[x_{0},(A-\mu I)^{s} y\right]=0, \quad y \in \mathscr{L}(\mu) .
$$

С другой стороны, из равенства (5) имеем

$$
\left[\left(A^{*}-\mu I\right)^{s} x_{0}, y\right]=(-\mu)^{s}\left[x_{0}, y\right] .
$$

Сравнивая $(10)$ и $(11)$, получаем $\left[x_{0}, y\right]=0$ при $y \in \mathscr{L}(\mu)$.

Таким образом, $x_{0} \in$ (1.h. $\left.\{\mathscr{L}(\mu) \mid \mu \in \sigma(A)\}\right)^{\perp}$. Из равенства $\mathscr{E}^{n}=$ l.h. $\{\mathscr{L}(\mu) \mid \mu \in$ $\sigma(A)\}$ следует, что $x_{0}=\theta$. Получили противоречие. 
СлеДСТвИЕ. Каждое подпространство $\mathscr{L}(\mu), \mu \in \sigma(A)$, невырождено.

Доказательство непосредственно следует из теоремы 1 , если в качестве $\mathscr{M}$ взять корневое подпространство $\mathscr{L}(\mu), \mu \in \sigma(A)$.

Tеорема 2. B $\mathscr{E}^{n}$ существует $A$-жорданов базис такой, что линейная оболочка любого набора максимальных жордановых чепочек, входящих в него, невырождена.

ДокАЗАТЕЛЬСТВо. В силу следствия и теоремы 1 без ограничения общности можно считать спектр оператора $A$ состоящим из одной точки $\lambda=0$ и все максимальные $A$-жордановы цепочки имеющими одинаковую длину $m$, т.е. произвольньй базис $(1)$ принимает вид $\left\{e_{i, j}\right\}, i=1,2, \ldots, k, j=1,2, \ldots, m$. Рассмотрим форму $\left[A^{m-1} x, x\right]$. В $\mathscr{E}^{n}$ существует вектор $e_{1, m}$ такой, что $\left[A^{m-1} e_{1, m}, e_{1, m}\right] \neq 0$. В самом деле, в противном случае $A^{m-1} x=0$ при всех $x \in \mathscr{E}^{n}$, а это противоречит тому, что длины всех максимальных жордановых цепочек равны $m$. Без ограничения обшности можно считать вектор $e_{1, m}$ выбранньц так, что $\left[A^{m-1} e_{1, m}, e_{1, m}\right]=a_{1}$, где $a_{1}=1$ или $a_{1}=-1$. Последовательность $\left\{e_{1, j}\right\}_{j=1}^{m}$ с $e_{1, j}=A^{m-j} e_{1, m}, j=1,2, \ldots, m$, является максимальной $A$-жордановой цепочкой. Согласно лемме 1 существует вектор $f_{1, m}$ такой, что выполнено равенство (2), а именно $A^{* m-1} f_{1, m}=A^{m-1} e_{1, m}=e_{1,1}$.

Заметим, что

$\left[A^{* m-1} f_{1, m}, f_{1, m}\right]=\left[A^{m-1} e_{1, m}, f_{1, m}\right]=\left[e_{1, m}, A^{* m-1} f_{1, m}\right]=\left[e_{1, m}, A^{m-1} e_{1, m}\right]=a_{1}$.

Из предположений относительно длин жордановых цепочек получаем, что последовательность $\left\{f_{1, j}\right\}_{j=1}^{m} \mathrm{c} f_{1, j}=A^{m-j} f_{1, m}, j=1,2, \ldots, m$, является максимальной $A^{*}$-жордановой цепочкой. Из выбора $A$ - и $A^{*}$-жордановых цепочек и леммы 2 следует невырожденность подпространств l.h. $\left\{e_{1, j}\right\}_{j=1}^{m}$ и l.h. $\left\{f_{1, j}\right\}_{j=1}^{m}$. Поэтому невырождено и $A$-инвариантное подпространство $\mathscr{E}^{n-m}=\left(\text { l.h. }\left\{f_{1, j}\right\}_{j=1}^{m}\right)^{\perp}$. Следовательно, в $\mathscr{E}^{n-m}$ сушествует вектор $e_{2, m}$ такой, что $\left[A^{m-1} e_{2, m}, e_{2, m}\right]=a_{2}$, где $a_{2}=1$ или $a_{2}=-1$. По тем жеправилам, что и вьше, построим $A$-жорданову цепочку $\left\{e_{2, j}\right\}_{j=1}^{m}$ и $A^{*}$-жорданову цепочку $\left\{f_{2, j}\right\}_{j=1}^{m}$. Тогда $\left[A^{m-1} e_{1, m}, e_{2, m}\right]=\left[A^{* m-1} f_{1, m}, f_{2, m}\right]=0$. Продолжив этот процесс, выделим в $\mathscr{E}^{n} A$-жорданов базис $\left\{e_{i, j}\right\}_{j=1}^{m}, i=1,2, \ldots, k$, и $A^{*}$-жорданов базис $\left\{f_{i, j}\right\}_{j=1}^{m}, i=1,2, \ldots, k$, такие, что

$$
\begin{gathered}
f_{i, 1}=A^{* m-1} f_{i, m}=A^{m-1} e_{i, m}=e_{i, 1}, \quad i=1,2, \ldots, k, \\
{\left[A^{m-1} e_{i, m}, e_{i, m}\right]=\left[A^{* m-1} f_{i, m}, f_{i, m}\right]=a_{i}, \quad a_{i}= \pm 1, \quad i=1,2, \ldots, k,} \\
{\left[A^{m-1} e_{i, m}, e_{s, m}\right]=\left[A^{* m-1} f_{i, m}, f_{s, m}\right]=0, \quad i \neq s, \quad i, s=1,2, \ldots, k .}
\end{gathered}
$$

Пусть $\left\{i_{r}\right\}_{r=1}^{s}-$ подмножество множества $\{i\}_{i=1}^{s}$. Для доказательства теоремы достаточно проверить, что l.h. $\left\{e_{i_{r}, j} \mid j=1,2, \ldots, m, r=1,2, \ldots, s\right\}$ - невырожденное подпространство. Если это не так, то согласно лемме 2 среди изотропных векторов есть вектор вида

$$
\sum_{r=1}^{s} \alpha_{r} e_{i_{r}, 1}
$$

Из (12) и (14) имеем $\alpha_{r}\left[e_{i_{r}, 1}, e_{i_{r}, m}\right]=0$, а из (13) получаем $\alpha_{r}=0$. 
2. Максимальные семидефинитные $A$-инвариантные подпространства. Целью этого пункта является алгебраическое доказательство существования максимальных семидефинитных $A$-инвариантных подпространств. Как известно (см., например, [3, с. 15]), размерность максимального неотрищательного (соответственно неположительного) подпространства не зависит от его выбора и совпадает с количеством положительных (соответственно отрищательных) собственных значений оператора $H$ с учетом кратности. Поэтому неотрицательное $\mathscr{M}^{+}$и неположительное $\mathscr{M}^{-} A$-инвариантные подпространства будут максимальными тогда и только тогда, когда

$$
\operatorname{dim} \mathscr{M}^{+}+\operatorname{dim} \mathscr{M}^{-}=n .
$$

Теорема 3 [10], [11]. Пусть $A$ есть $H$-диссипативный оператор в $n$-мерном $H$-пространстве $\mathscr{E}^{n}$. Тогда существуют А-инвариантные максимальное неотрииательное $\mathscr{M}^{+}$и максимальное неположсительное $\mathscr{M}^{-}$подпространства такие, что $\operatorname{Im} \sigma\left(\left.A\right|_{\mathscr{M}^{+}}\right) \geqslant 0 u \operatorname{Im} \sigma\left(\left.A\right|_{\mathscr{M}^{-}}\right) \leqslant 0$.

ДоказАТЕЛЬСтво. Рассмотрим разложение пространства $\mathscr{E}^{n}=\mathscr{E}_{1}^{+}+\mathscr{E}_{1}^{-}+\mathscr{E}_{1}$, где $\mathscr{E}_{1}^{+}$- линейная оболочка корневых векторов оператора $A$, соответствующих $\lambda \in \sigma_{p}(A)$, $\operatorname{Im} \lambda>0, \mathscr{E}_{1}^{-}$- линейная оболочка корневых векторов оператора $A$, соответствующих $\lambda \in \sigma_{p}(A), \operatorname{Im} \lambda<0$, и $\mathscr{E}_{1}-$ линейная оболочка корневых векторов оператора $A$, соответствующих вещественньп $\lambda \in \sigma_{p}(A)$. Из [14] (см. также [1, с. 123-124]) следует неотрицательность $\mathscr{E}_{1}^{+}$, неположительность $\mathscr{E}_{1}^{-}$и невырожденность $\mathscr{E}_{1}$. Поэтому достаточно указать максимальные в $\mathscr{E}_{1}$ неотрицательное инвариантное подпространство $\mathscr{M}_{2}^{+}$ и неположительное инвариантное подпространство $\mathscr{M}_{2}^{-}, H$-ортогональные $\mathscr{E}_{1}^{+}$и $\mathscr{E}_{1}^{-}$.

Пусть $(1)$ - базис в $\mathscr{E}_{1}$ из жордановых цепочек оператора $\left.A\right|_{\mathscr{E}_{1}}$, построенньй по методу, изложенному в доказательстве теоремы 2. Рассмотрим подпространства

$$
\begin{gathered}
\mathscr{M}_{1}^{0}=\text { l.h. }\left\{e_{i, j_{i}} \mid j_{i}=1,2, \ldots, \frac{n_{i}}{2}, i: n_{i} \text { четно }\right\}, \\
\mathscr{M}_{1}^{+}=\text {l.h. }\left\{e_{i, j_{i}} \mid j_{i}=1,2, \ldots, \frac{n_{i}+1}{2}, i: n_{i} \text { нечетно и }\left[e_{i, 1}, e_{i, n_{i}}\right]=1\right\}, \\
\mathscr{M}_{1}^{-}=\text {l.h. }\left\{e_{i, j_{i}} \mid j_{i}=1,2, \ldots, \frac{n_{i}+1}{2}, i: n_{i} \text { нечетно и }\left[e_{i, 1}, e_{i, n_{i}}\right]=-1\right\} .
\end{gathered}
$$

С помощью формулы [14] (см. также [1, с. 125])

$\left(A^{*}-\alpha I\right)^{l-p}(A-\alpha I)^{p} x=0, \quad$ если $\alpha=\bar{\alpha},(A-\alpha I)^{l} x=0$ и $p=\left[\frac{l}{2}\right],\left[\frac{l}{2}\right]+1, \ldots, l-1$,

можно доказать, что $\mathscr{M}_{1}^{0}-$ нейтральное, $\mathscr{M}_{1}^{+}$- положительное, $\mathscr{M}_{1}^{-}$- отрицательное подпространства, они попарно $H$-ортогональны и $H$-ортогональны $\mathscr{E}_{1}^{+}+\mathscr{E}_{1}^{-}$. Поэтому $\mathscr{M}_{2}^{+}=\mathscr{M}_{1}^{0}+\mathscr{M}_{1}^{+}$и $\mathscr{M}_{2}^{-}=\mathscr{M}_{1}^{0}+\mathscr{M}_{1}^{-}$- искомые неотрицательное и неположительное инвариантные подпространства, максимальность которых в $\mathscr{E}_{1}$ следует из (15).

Авторы признательны А. А. Шкаликову за полезные обсуждения. 


\section{СПИСОК ЦИТИРОВАННОЙ ЛИТЕРАТУРЫ}

[1] Азизов Т.Я., Иохвидов И. С. Основы теории линейных операторов в пространствах с индефинитной метрикой. М.: Наука, 1986.

[2] Мальцев А.И. Основы линейной алгебры. М.-Л.: ГИТТЛ, 1948.

[3] Gohberg I., Lancaster P., Rodman L. Matrices and Indefinite Scalar Products. Basel: Birkhäuser, 1983.

[4] Shkalikov A. A. Operator pencils arising in elasticity and hydrodynamics: the instability index formula // Operator Theory: Advances and Applications. V. 87. Basel: Birkhäuser, 1996. P. 258-285.

[5] Азизов Т.Я., Сухочёва Л.И. Новый подход к доказательству теоремы о приведении матриц к жордановой форме // Функциональный анализ. Линейные пространства. Ульновск, 1990. C. $3-5$.

[6] Костюченко А. Г., Шкаликов А. А. Самосопряженные операторные пучки и эллиптические задачи // Функцион. анализ и его прилож. 1983. Т. 17. № 2. С. 38-61.

[7] Шкаликов А. А. О принципах отбора и свойствах части собственных и присоединенных элементов пучков операторов // Вестн. МГУ. Сер. 1. Матем., мех. 1988. №4. С. 16-25.

[8] Шкаликов А. А. Эллиптические уравнения в гильбертовом пространстве и связанные спектральные задачи // Тр. семинара им. И.Г. Петровского. 1989. Т. 14. С. 140-224.

[9] Azizov T. Ya., Binding P. A., Bognar J., Najman B. Nondegenerate Jordan subspaces of self-adjoint operators in indefinite spaces // Linear Algebra Appl. 1994. V. 207. P. $37-48$.

[10] Азизов Т.Я. Инвариантные подпространства и критерии полноты системы корневых векторов $J$-диссипативных операторов в пространстве Понтрягина $\Pi_{\kappa} / /$ Докл. АН СССР. 1971. T. 200. № 5. C. 1015-1017.

[11] Крейн М.Г., Лангер Г. О дефинитных подпространствах и обобщенных резольвентах эрмитова оператора в пространстве $\Pi_{\kappa} / /$ Функцион. анализ и его прилож. 1971. Т. 5. № 7 . C. $59-71$.

[12] Ran A.C. M., Temme D. Dissipative matrices and invariant maximal semidefinite subspaces // Linear Algebra Appl. 1994. V. 212/213. P. 169-213.

[13] Azizov T. Ya., Langer H. Some spectral properties of contractive and expansive operators in indefinite inner product spaces // Math. Nachr. 1993. V. 162. P. 247-259.

[14] Азизов Т. Я. Диссипативные операторы в гильертовом пространстве с индефинитной метрикой // Изв. АН СССР. Сер. матем. 1973. Т. 37. № 3. С. 639-662. 